Proceedings of the Edinburgh Mathematical Society (2003) 46, 513-529 (C)

DOI:10.1017/S0013091502000809 Printed in the United Kingdom

\title{
THE MAXIMAL ORDER PROPERTY FOR QUANTUM DETERMINANTAL RINGS
}

\author{
T. H. LENAGAN ${ }^{1}$ AND L. RIGAL ${ }^{2}$ \\ ${ }^{1}$ School of Mathematics, University of Edinburgh, \\ James Clerk Maxwell Building, King's Buildings, \\ Mayfield Road, Edinburgh EH9 3JZ, UK (tom@maths.ed.ac.uk) \\ ${ }^{2}$ Université Jean Monnet (Saint-Étienne), Faculté des Sciences et Techniques, \\ Département de Mathématiques, 23 rue du Docteur Paul Michelon, \\ 42023 Saint-Étienne Cédex 2, France
}

(Laurent.Rigal@univ-st-etienne.fr)

(Received 26 August 2002)

\begin{abstract}
We develop a method of reducing the size of quantum minors in the algebra of quantum matrices $\mathcal{O}_{q}\left(M_{n}\right)$. We use the method to show that the quantum determinantal factor rings of $\mathcal{O}_{q}\left(M_{n}(\mathbb{C})\right.$ ) are maximal orders, for $q$ an element of $\mathbb{C}$ transcendental over $\mathbb{Q}$.
\end{abstract}

Keywords: quantum matrices; quantum minors; quantum determinantal rings; maximal orders

2000 Mathematics subject classification: Primary 16P40; 16W35; $20 \mathrm{G} 42$

\section{Introduction}

Throughout, $\mathbb{K}$ will denote a base field, $q$ a non-zero element of $\mathbb{K}$, and $m, n$ positive integers. We denote by $\mathcal{O}_{q}\left(M_{m, n}\right)$ the quantization of the ring of regular functions on $m \times n$ matrices with entries in $\mathbb{K}$; it is the $\mathbb{K}$-algebra generated by $m n$ indeterminates $X_{i j}, 1 \leqslant i \leqslant m$ and $1 \leqslant j \leqslant n$, subject to the following relations:

$$
\left.\begin{array}{rl}
X_{i j} X_{i l} & =q X_{i l} X_{i j}, \\
X_{i j} X_{k j} & =q X_{k j} X_{i j}, \\
X_{i l} X_{k j} & =X_{k j} X_{i l}, \\
X_{i j} X_{k l}-X_{k l} X_{i j} & =\left(q-q^{-1}\right) X_{i l} X_{k j}
\end{array}\right\}
$$

for $1 \leqslant i<k \leqslant m$ and $1 \leqslant j<l \leqslant n$.

It will be convenient to use the following notation: setting

$$
\boldsymbol{X}=\left(X_{i j}\right)_{1 \leqslant i \leqslant m, 1 \leqslant j \leqslant n}
$$

we will denote $\mathcal{O}_{q}\left(M_{m, n}\right)$ by $\mathbb{K}_{q}[\boldsymbol{X}]$. 
Let $A$ be any $\mathbb{K}$-algebra, we say that an $m \times n$ matrix $\left(a_{i j}\right)$ with entries in $A$ is a $q$ quantum matrix if the map $X_{i j} \mapsto a_{i j}$ induces a homomorphism of algebras $\mathbb{K}_{q}[\boldsymbol{X}] \rightarrow A$. If, in addition, this homomorphism is a monomorphism, then we say that the matrix $\left(a_{i j}\right)$ is a generic q-quantum matrix; clearly, $\boldsymbol{X}$ is a generic q-quantum matrix, by definition.

In the case where $m=n$, the quantum determinant of $\boldsymbol{X}$ is defined by

$$
\operatorname{det}_{q}=\operatorname{det}_{q} \boldsymbol{X}:=\sum_{\sigma \in S_{n}}(-q)^{l(\sigma)} X_{1, \sigma(1)} \cdots X_{n, \sigma(n)}
$$

from [9, Theorem 4.6.1], we know that $\operatorname{det}_{q}$ is in the centre of $\mathcal{O}_{q}\left(M_{n}\right):=\mathcal{O}_{q}\left(M_{n, n}\right)$. Clearly, if $I \subseteq \mathbb{N}_{m}:=\{1, \ldots, m\}$ and $J \subseteq \mathbb{N}_{n}:=\{1, \ldots, n\}$ with $|I|=|J|=t$, that is, $I$ and $J$ both have $t$ elements, then the submatrix obtained from $\boldsymbol{X}$ by keeping the rows indexed by elements of $I$ and the columns indexed by elements of $J$ is a generic $q$-quantum matrix; and so we can speak of its quantum determinant. Such an element is called a $t \times t$ quantum minor of $\boldsymbol{X}$ and is denoted by $[I \mid J]$. In order to simplify the notation, if $I$ and $J$ are given by the explicit list of their elements, $I=\left\{i_{1}<\cdots<i_{t}\right\}$ and $J=\left\{j_{1}<\cdots<j_{t}\right\}$, we will use the notation $\left[i_{t}, \ldots, i_{1} \mid j_{1}, \ldots, j_{t}\right]$. It follows at once from the above that

$$
\text { for } I \subseteq \mathbb{N}_{m}, J \subseteq \mathbb{N}_{n} \text {, with }|I|=|J|=t, i \in I \text { and } j \in J, \quad[I \mid J] X_{i j}=X_{i j}[I \mid J]
$$

We need to use several identities involving quantum minors. Many of these are obtained from $[\mathbf{9}]$. However, it is worth noting that the conventions used in $[\mathbf{9}]$ are slightly different from ours. In order to make the notation fit we must replace $q$ by $q^{-1}$ each time we use a relation from [9]. For convenience, some of the identities we use are collected in an appendix.

For any positive integer $t$ such that $t \leqslant \min \{m, n\}$, we denote by $\mathcal{I}_{t}(\boldsymbol{X})$ the two-sided ideal of $\mathbb{K}_{q}[\boldsymbol{X}]$ generated by the $t \times t$ quantum minors of $\boldsymbol{X}$; such ideals will be referred to as quantum determinantal ideals. For each such $t$, we define the quantum determinantal ring $R_{t}(\boldsymbol{X}):=\mathbb{K}_{q}[\boldsymbol{X}] / \mathcal{I}_{t}(\boldsymbol{X})$. It has been proved in $[\mathbf{1}]$ that $R_{t}(\boldsymbol{X})$ is a domain. Here, we are interested in the question of whether or not $R_{t}(\boldsymbol{X})$ is a maximal order in its division ring of fractions. This has already been established for $R_{2}(\boldsymbol{X})$ in [10]. We will show that the localized ring $R_{t}(\boldsymbol{X})\left[x_{1 n}^{-1}\right]$, where $x_{1 n}:=X_{1 n}+\mathcal{I}_{t}(\boldsymbol{X})$, is a maximal order for each $t$, and we are then able to deduce that $R_{t}(\boldsymbol{X})$ is a maximal order, in the case where $\mathbb{K}=\mathbb{C}$ and $q$ is an element of $\mathbb{C}$ transcendental over $\mathbb{Q}$. The question for general $\mathbb{K}$ and any $q$ remains open: we conjecture that all quantum determinantal rings are maximal orders. The problem is a technical one: it is necessary to show that a certain ideal is a prime ideal. The restriction to $q$ an element of $\mathbb{C}$ transcendental over $\mathbb{Q}$ is because the relevant ideal is shown to be prime in $[\mathbf{6}]$ for this case. We can answer the question for general $0 \neq q \in \mathbb{K}$ for the case of $R_{n}(\boldsymbol{X})\left(=\mathcal{O}_{q}\left(M_{n}\right) /\left\langle\operatorname{det}_{q}\right\rangle\right)$, when $\boldsymbol{X}$ is $n \times n$, and also for the case $R_{3}(\boldsymbol{X})$ for general $m \times n$. A proof of the former case is included in this paper since it is relatively short, and of independent interest. The proof of the latter is not given here, since it is somewhat ad hoc and rather long. 


\section{A new set of generators for $\mathbb{K}_{q}[X]_{X_{1 n}}$}

It follows at once from (1.1) that $X_{1 n}$ is a normal element of $\mathbb{K}_{q}[\boldsymbol{X}]$. So, $\left\{X_{1 n}^{i}, i \in \mathbb{N}\right\}$ is a right denominator set of $\mathbb{K}_{q}[\boldsymbol{X}]$; the corresponding localization of $\mathbb{K}_{q}[\boldsymbol{X}]$ will be denoted $\mathbb{K}_{q}[\boldsymbol{X}]_{X_{1 n}}$.

For the remainder of this section we assume that $\min \{m, n\} \geqslant 2$. In $\mathbb{K}_{q}[\boldsymbol{X}]_{X_{1 n}}$, we consider the following elements, for $2 \leqslant i \leqslant m$ and $1 \leqslant j \leqslant n-1$ :

$$
X_{i j}^{\prime}=X_{i j}-q^{-1} X_{1 j} X_{i n} X_{1 n}^{-1}=-q^{-1}[i, 1 \mid j, n] X_{1 n}^{-1} .
$$

Set

$$
\tilde{\boldsymbol{X}}=\left(\begin{array}{cccc}
X_{11} & \ldots & X_{1, n-1} & X_{1 n} \\
X_{21}^{\prime} & \ldots & X_{2, n-1}^{\prime} & X_{2 n} \\
\vdots & & \vdots & \vdots \\
X_{m 1}^{\prime} & \ldots & X_{m, n-1}^{\prime} & X_{m n}
\end{array}\right) \quad \text { and } \quad \boldsymbol{X}^{\prime}=\left(\begin{array}{ccc}
X_{21}^{\prime} & \ldots & X_{2, n-1}^{\prime} \\
\vdots & & \vdots \\
X_{m 1}^{\prime} & \ldots & X_{m, n-1}^{\prime}
\end{array}\right)
$$

with the convention that the index of a row in $\boldsymbol{X}^{\prime}$ is actually the index of the corresponding row in $\tilde{\boldsymbol{X}}$. So, for instance, $X_{21}^{\prime} \cdots X_{2, n-1}^{\prime}$ is referred to as the row of index 2 in $\boldsymbol{X}^{\prime}$.

It is clear that the entries of $\tilde{\boldsymbol{X}}$ together with $X_{1 n}^{-1}$ form a new set of algebra generators for $\mathbb{K}_{q}[\boldsymbol{X}]_{X_{1 n}}$. Our next aim is to give a description of $\mathbb{K}_{q}[\boldsymbol{X}]_{X_{1 n}}$ based on this set of generators.

In order to do this, we need to know the relations between the entries of the matrix $\tilde{\boldsymbol{X}}$. This problem is dealt with in Lemma 2.1 and Proposition 2.2, where we obtain a set of relations. We will show later that the relations obtained in these results give the complete set of relations between entries of $\tilde{\boldsymbol{X}}$.

Lemma 2.1. The matrix $\boldsymbol{X}^{\prime}$ is a q-quantum matrix, and all its entries commute with $X_{1 n}$.

Proof. Let $2 \leqslant i, k \leqslant m$ and $1 \leqslant j, l \leqslant n-1$. From (1.2) we see that $X_{1 n}$ commutes with the $2 \times 2$ quantum minor $[i, 1 \mid j, n]$. So $X_{i j}^{\prime} X_{1 n}=X_{1 n} X_{i j}^{\prime}$ follows from the second equality of (2.1). Moreover, from (2.1) again, we have $X_{i j}^{\prime}=-q^{-1}[i, 1 \mid j, n] X_{1 n}^{-1}$ and $X_{k l}^{\prime}=-q^{-1}[k, 1 \mid l, n] X_{1 n}^{-1}$. The desired relations between $X_{i j}^{\prime}$ and $X_{k l}^{\prime}$ are deduced in an obvious manner from [9, Theorem 5.2.1] applied to the submatrix of $\boldsymbol{X}$ defined by rows $1, i, k$ and columns $j, l, n$.

The following proposition is a list of relations between entries of $\tilde{\boldsymbol{X}}$. Relations between two elements of the first row or of the last column of $\tilde{\boldsymbol{X}}$ are deduced from the fact that $\boldsymbol{X}$ is a $q$-quantum matrix.

Proposition 2.2. The following relations hold between entries of the matrix $\tilde{\boldsymbol{X}}$.

(1) If $1 \leqslant j \leqslant n-1$ and $2 \leqslant i \leqslant m$, then

$$
X_{1 j} X_{i n}-q^{2} X_{i n} X_{1 j}=q\left(q^{2}-1\right) X_{i j}^{\prime} X_{1 n}
$$


(2.1) For $1 \leqslant j \leqslant n-1$ and $2 \leqslant k \leqslant m$,

$$
\begin{array}{rlrl}
X_{1 j} X_{k l}^{\prime}-X_{k l}^{\prime} X_{1 j} & =\left(q^{-1}-q\right) X_{1 l} X_{k j}^{\prime}, & & \text { for } 1 \leqslant l \leqslant j-1, \\
X_{1 j} X_{k j}^{\prime} & =q^{-1} X_{k j}^{\prime} X_{1 j}, & & \\
X_{1 j} X_{k l}^{\prime} & =X_{k l}^{\prime} X_{1 j}, & \text { for } j+1 \leqslant l \leqslant n-1 .
\end{array}
$$

(2.2) For $2 \leqslant i \leqslant m$ and $1 \leqslant l \leqslant n-1$,

$$
\begin{aligned}
X_{i n} X_{k l}^{\prime} & =X_{k l}^{\prime} X_{i n}, & & \text { for } 2 \leqslant k \leqslant i-1, \\
X_{i n} X_{i l}^{\prime} & =q X_{i l}^{\prime} X_{i n}, & & \\
X_{i n} X_{k l}^{\prime}-X_{k l}^{\prime} X_{i n} & =\left(q-q^{-1}\right) X_{k n} X_{i l}^{\prime}, & & \text { for } i+1 \leqslant k \leqslant m .
\end{aligned}
$$

(3) If $1 \leqslant k<l \leqslant n$ and $1 \leqslant i<j \leqslant m$, then

$$
X_{1 k} X_{1 l}=q X_{1 l} X_{1 k} \quad \text { and } \quad X_{i n} X_{j n}=q X_{j n} X_{i n} .
$$

Proof. Part (3) is obvious. The other relations are all obtained in the following way. First, use the definition (2.1) to translate the desired relation into a new one that uses only the entries of $\boldsymbol{X}$, by multiplying by $X_{1 n}$. The relation so obtained involves entries coming from a certain submatrix of $\boldsymbol{X}$ of size at most $3 \times 3$. Use relation (A 1) and relations (A 2) to check that the relation holds. Since these are easy but tedious computations we omit the details.

The next thing we want to do is to show that $\boldsymbol{X}^{\prime}$ is a generic $q$-quantum matrix. We use a computation with Gelfand-Kirillov dimension (GKdim) to do this. The following lemma is probably well known, but we have not located the exact statement that we need; and so we include a proof.

Lemma 2.3. Let $B$ be a $\mathbb{K}$-algebra. Suppose $A$ is a subalgebra of $B$ and $x$ is an element of $B$ such that $B$ is generated by $A$ and $x$ as an algebra. Furthermore, suppose there exists a finite-dimensional subspace $V$ of $A$ that generates $A$ as an algebra and such that $x V \subseteq V x+A$. Then $\operatorname{GKdim}(B) \leqslant \operatorname{GKdim}(A)+1$.

Proof. Without loss of generality, we may assume that $1 \in V$. We denote by $W$ the subspace of $B$ spanned by $x$ and $V$. Thus, $W$ is a finite-dimensional subspace of $B$ that generates $B$ as an algebra. Since $x V \subseteq V x+A$ and $V$ is finite dimensional, there exists $m \in \mathbb{N}^{*}$ such that $x V \subseteq V x+V^{m}$. An easy induction then shows that, for $n \in \mathbb{N}$, $x V^{n} \subseteq V^{n} x+V^{m+n}$.

We claim that

$$
W^{n} \subseteq V^{n m}+V^{n m} x+\cdots+V^{n m} x^{n},
$$

for all $n \in \mathbb{N}$. The inclusion (2.2) is trivial for $n=0$ (recall the standard convention that $\left.V^{0}=W^{0}=\mathbb{K}\right)$. Now, assume $(2.2)$ holds for $p \in \mathbb{N}$, that is $W^{p} \subseteq V^{p m}+V^{p m} x+\cdots+$ $V^{p m} x^{p}$. It follows that

$$
\begin{aligned}
x W^{p} & \subseteq x V^{p m}+x V^{p m} x+\cdots+x V^{p m} x^{p} \\
& \subseteq V^{p m} x+V^{p m+m}+V^{p m} x^{2}+V^{p m+m} x+\cdots+V^{p m} x^{p+1}+V^{p m+m} x^{p} .
\end{aligned}
$$


So $x W^{p} \subseteq V^{m(p+1)}+\cdots+V^{m(p+1)} x^{p+1}$. On the other hand, $V W^{p} \subseteq V^{m p+1}+\cdots+$ $V^{m p+1} x^{p}$. We finally obtain

$$
W^{p+1}=(V+\mathbb{K} x) W^{p} \subseteq V^{m(p+1)}+\cdots+V^{m(p+1)} x^{p+1} .
$$

This establishes (2.2) by induction on $n$.

From (2.2), it follows that $\operatorname{dim} W^{n} \leqslant(n+1) \operatorname{dim} V^{m n}$, for all $n \in \mathbb{N}$, and thus

$$
\varlimsup \log _{n} \operatorname{dim} W^{n} \leqslant \varlimsup \log _{n} \operatorname{dim} V^{m n}+1 .
$$

This establishes that $\operatorname{GK} \operatorname{dim} B \leqslant \operatorname{GK} \operatorname{dim} A+1$.

Proposition 2.4. The matrix $\boldsymbol{X}^{\prime}$ is a generic q-quantum matrix.

Proof. In this proof, we denote by $A$ the subalgebra of $\mathbb{K}_{q}[\boldsymbol{X}]_{X_{1 n}}$ generated by the entries of $\boldsymbol{X}^{\prime}$. By Lemma 2.1, there is a surjective morphism $\phi: \mathcal{O}_{q}\left(M_{m-1, n-1}\right) \rightarrow$ $A$. We want to prove that $\operatorname{ker} \phi=\langle 0\rangle$. Assume that this is not the case. Then, since $\mathcal{O}_{q}\left(M_{m-1, n-1}\right)$ is a domain, $\operatorname{ker} \phi$ must contain a regular element of $\mathcal{O}_{q}\left(M_{m-1, n-1}\right)$ and thus we have $\operatorname{GKdim} A<\operatorname{GKdim} \mathcal{O}_{q}\left(M_{m-1, n-1}\right)=(m-1)(n-1)$.

By Lemma 2.1, we see that the subalgebra $B$ of $\mathbb{K}_{q}[\boldsymbol{X}]_{X_{1 n}}$ generated by $A$ and $X_{1 n}$ is an extension of $A$ of the type investigated in Lemma 2.3. Thus, we must have $\operatorname{GKdim} B<(m-1)(n-1)+1$. Moreover, since $X_{1 n}$ is central in $B$, [4, Proposition 4.2] shows that $\operatorname{GKdim} B_{X_{1 n}}<(m-1)(n-1)+1$. Now, it is clear that $\mathbb{K}_{q}[\boldsymbol{X}]_{X_{1 n}}$ can be obtained by successive algebra extensions starting from $B_{X_{1 n}}$ and adding (in this order) $X_{11}, \ldots, X_{1, n-1}$ and then $X_{m n}, \ldots, X_{2 n}$ (this is because $\mathbb{K}_{q}[\boldsymbol{X}]_{X_{1 n}}$ is generated by the entries of $\tilde{\boldsymbol{X}}$ and $\left.X_{1 n}^{-1}\right)$. Moreover, Proposition 2.2 shows that at each step, the extension is of the type investigated in Lemma 2.3. Thus, $(m-1)+(n-1)$ applications of this lemma show that we must have

$$
\operatorname{GKdim} \mathbb{K}_{q}[\boldsymbol{X}]_{X_{1 n}}<(m-1)(n-1)+1+(m-1)+(n-1)=m n .
$$

However, we know that GKdim $\mathbb{K}_{q}[\boldsymbol{X}]_{X_{1 n}} \geqslant \operatorname{GKdim} \mathbb{K}_{q}[\boldsymbol{X}]=m n$. This is a contradiction and thus we have proved that $\operatorname{ker} \phi=\langle 0\rangle$.

Proposition 2.4 states that the subalgebra of $\mathbb{K}_{q}[\boldsymbol{X}]_{X_{1 n}}$ generated by $\boldsymbol{X}^{\prime}$ is isomorphic to $\mathcal{O}_{q}\left(M_{m-1, n-1}\right)$; for this reason, we denote it by $\mathbb{K}_{q}\left[\boldsymbol{X}^{\prime}\right]$.

The following remark will be useful in what follows.

Remark 2.5. We denote by $\mathrm{F}\left\langle X_{1}, \ldots, X_{p}\right\rangle$ the free $\mathbb{K}$-algebra on $p$ generators $X_{1}, \ldots, X_{p}$. Let $I$ be the ideal generated by elements $f_{1}, \ldots, f_{s} \in \mathrm{F}\left\langle X_{1}, \ldots, X_{p}\right\rangle$, and set $A:=\mathrm{F}\left\langle X_{1}, \ldots, X_{p}\right\rangle / I$. Finally, let $\sigma$ be an automorphism of $\mathrm{F}\left\langle X_{1}, \ldots, X_{p}\right\rangle$ and $\delta$ be a left $\sigma$-derivation of $\mathrm{F}\left\langle X_{1}, \ldots, X_{p}\right\rangle$ such that $\sigma(I)=I$ and $\delta(I) \subseteq I$. We denote by $\bar{\sigma}$ the automorphism of $A$ induced by $\sigma$ and by $\bar{\delta}$ the left $\bar{\sigma}$-derivation of $A$ induced by $\delta$. Then $A[x ; \bar{\sigma}, \bar{\delta}]$ is isomorphic to the algebra $F\left\langle X_{1}, \ldots, X_{p}, X\right\rangle / J$, where $J$ is the ideal of $F\left\langle X_{1}, \ldots, X_{p}, X\right\rangle$ generated by $f_{1}, \ldots, f_{s}$ and the $p$ elements $X X_{i}-\sigma\left(X_{i}\right) X-\delta\left(X_{i}\right)$ (here we identify $\mathrm{F}\left\langle X_{1}, \ldots, X_{p}\right\rangle$ and the subalgebra of $\mathrm{F}\left\langle X_{1}, \ldots, X_{p}, X\right\rangle$ generated by $\left.X_{1}, \ldots, X_{p}\right)$. 
We now proceed to show that $\mathbb{K}_{q}[\boldsymbol{X}]_{X_{1 n}}$ can be described as an iterated Ore extension of a Laurent polynomial extension of its subalgebra $\mathbb{K}_{q}\left[\boldsymbol{X}^{\prime}\right]$ (recall the notation we fixed immediately after Proposition 2.4). For this, we first construct an algebra $\mathcal{B}$ which is an iterated Ore extension of $\mathcal{O}_{q}\left(M_{m-1, n-1}\right)$. We then show that $\mathcal{B}$ is isomorphic to $\mathbb{K}_{q}[\boldsymbol{X}]_{X_{1 n}}$.

Let us start with the algebra $\mathcal{O}_{q}\left(M_{m-1, n-1}\right)$. For convenience of notation, we denote its canonical generators by $Y_{i j}^{\prime}$ for $2 \leqslant i \leqslant m$ and $1 \leqslant j \leqslant n-1$. Thus,

$$
\boldsymbol{Y}^{\prime}=\left(Y_{i j}^{\prime}\right)_{2 \leqslant i \leqslant m, 1 \leqslant j \leqslant n-1}
$$

is a generic $q$-quantum matrix and, following our previous notation, we have

$$
\mathcal{O}_{q}\left(M_{m-1, n-1}\right)=\mathbb{K}_{q}\left[\boldsymbol{Y}^{\prime}\right] .
$$

Now we consider the Laurent polynomial extension $\mathcal{A}=\mathbb{K}_{q}\left[\boldsymbol{Y}^{\prime}\right]\left[Y_{1 n}^{ \pm 1}\right]$ obtained from $\mathbb{K}_{q}\left[\boldsymbol{Y}^{\prime}\right]$ adding a central indeterminate, denoted by $Y_{1 n}$, and its inverse.

By Remark 2.5, we know how to describe $\mathcal{A}$ as a quotient of a free algebra. It is then easy to see that we can define a (unique) left skew derivation $\left(\sigma_{11}, \delta_{11}\right)$ of $\mathcal{A}$ such that $\delta_{11}=0$ and such that $\sigma_{11}\left(Y_{1 n}\right)=q Y_{1 n}$ and, for $2 \leqslant k \leqslant m$ and $2 \leqslant l \leqslant n-1$, $\sigma_{11}\left(Y_{k 1}^{\prime}\right)=q^{-1} Y_{k 1}^{\prime}$ and $\sigma_{11}\left(Y_{k l}^{\prime}\right)=Y_{k l}^{\prime}$. We put $\mathcal{A}_{11}=\mathcal{A}\left[Y_{11} ; \sigma_{11}, \delta_{11}\right]$.

Now, for $2 \leqslant p \leqslant n-1$, we construct an algebra $\mathcal{A}_{1 p}$ by induction on $p$. For $1 \leqslant p \leqslant$ $n-2$, by Remark 2.5 , we know how to describe $\mathcal{A}_{1 p}$ as a quotient of a free algebra. It is then easy to check that we can define a (unique) left skew derivation $\left(\sigma_{1, p+1}, \delta_{1, p+1}\right)$ of $\mathcal{A}_{1 p}$ such that, for $2 \leqslant k \leqslant m$,

$$
\begin{aligned}
& \sigma_{1, p+1}\left(Y_{k l}^{\prime}\right)=Y_{k l}^{\prime}, \quad \delta_{1, p+1}\left(Y_{k l}^{\prime}\right)=\left(q^{-1}-q\right) Y_{1 l} Y_{k, p+1}^{\prime} \quad \text { for } 1 \leqslant l \leqslant p, \\
& \sigma_{1, p+1}\left(Y_{k, p+1}^{\prime}\right)=q^{-1} Y_{k, p+1}^{\prime}, \quad \delta_{1, p+1}\left(Y_{k, p+1}^{\prime}\right)=0, \\
& \sigma_{1, p+1}\left(Y_{k l}^{\prime}\right)=Y_{k l}^{\prime}, \quad \delta_{1, p+1}\left(Y_{k l}^{\prime}\right)=0 \quad \text { for } p+2 \leqslant l \leqslant n-1, \\
& \sigma_{1, p+1}\left(Y_{1 n}\right)=q Y_{1 n}, \quad \delta_{1, p+1}\left(Y_{1 n}\right)=0, \\
& \sigma_{1, p+1}\left(Y_{1 l}\right)=q^{-1} Y_{1 l}, \quad \delta_{1, p+1}\left(Y_{1 l}\right)=0 \quad \text { for } 1 \leqslant l \leqslant p .
\end{aligned}
$$

Then, for $1 \leqslant p \leqslant n-2$, we put $\mathcal{A}_{1, p+1}=\mathcal{A}_{1, p}\left[Y_{1, p+1} ; \sigma_{1, p+1}, \delta_{1, p+1}\right]$.

Thus, we now have a first sequence of Ore extensions: $\mathcal{A}, \mathcal{A}_{11}, \ldots, \mathcal{A}_{1, n-1}$. To finish the construction of $\mathcal{B}$, we need a second such sequence, which we now define.

By Remark 2.5, we know how to describe $\mathcal{A}_{1, n-1}$ as a quotient of a free algebra. It is then easy to see that we can define a (unique) left skew derivation $\left(\sigma_{m n}, \delta_{m n}\right)$ of $\mathcal{A}_{1, n-1}$ such that, for $1 \leqslant l \leqslant n-1$,

$$
\begin{aligned}
& \sigma_{m n}\left(Y_{k l}^{\prime}\right)=Y_{k l}^{\prime}, \quad \delta_{m n}\left(Y_{k l}^{\prime}\right)=0, \quad \text { for } 2 \leqslant k \leqslant m-1, \\
& \sigma_{m n}\left(Y_{m l}^{\prime}\right)=q Y_{m l}^{\prime}, \quad \delta_{m n}\left(Y_{m l}^{\prime}\right)=0 \\
& \sigma_{m n}\left(Y_{1 n}\right)=q^{-1} Y_{1 n}, \quad \delta_{m n}\left(Y_{1 n}\right)=0 \\
& \sigma_{m n}\left(Y_{1 l}\right)=q^{-2} Y_{1 l}, \quad \delta_{m n}\left(Y_{1 l}\right)=\left(q^{-1}-q\right) Y_{m l}^{\prime} Y_{1 n} .
\end{aligned}
$$

We put $\mathcal{A}_{m n}=\mathcal{A}_{1, n-1}\left[Y_{m n} ; \sigma_{m n}, \delta_{m n}\right]$. 
Now, for $2 \leqslant s \leqslant m-1$, we construct an algebra $\mathcal{A}_{s n}$, by decreasing induction on $s$.

For $3 \leqslant s \leqslant m$, by Remark 2.5 , we know how to describe $\mathcal{A}_{s n}$ as a quotient of a free algebra. It is then easy to check that we can define a (unique) left skew derivation $\left(\sigma_{s-1, n}, \delta_{s-1, n}\right)$ of $\mathcal{A}_{s n}$ such that, for $1 \leqslant l \leqslant n-1$,

$$
\begin{aligned}
& \sigma_{s-1, n}\left(Y_{k l}^{\prime}\right)=Y_{k l}^{\prime}, \quad \delta_{s-1, n}\left(Y_{k l}^{\prime}\right)=0 \quad \text { for } 2 \leqslant k \leqslant s-2, \\
& \sigma_{s-1, n}\left(Y_{s-1, l}^{\prime}\right)=q Y_{s-1, l}^{\prime}, \quad \delta_{s-1, n}\left(Y_{s-1, l}^{\prime}\right)=0, \\
& \sigma_{s-1, n}\left(Y_{k l}^{\prime}\right)=Y_{k l}^{\prime}, \quad \delta_{s-1, n}\left(Y_{k l}^{\prime}\right)=\left(q-q^{-1}\right) Y_{k n} Y_{s-1, l}^{\prime} \quad \text { for } s \leqslant k \leqslant m, \\
& \sigma_{s-1, n}\left(Y_{1 n}\right)=q^{-1} Y_{1 n}, \quad \delta_{s-1, n}\left(Y_{1 n}\right)=0, \\
& \sigma_{s-1, n}\left(Y_{1 l}\right)=q^{-2} Y_{1 l}, \quad \delta_{s-1, n}\left(Y_{1 l}\right)=\left(q^{-1}-q\right) Y_{s-1, l}^{\prime} Y_{1 n}, \\
& \sigma_{s-1, n}\left(Y_{k n}\right)=q Y_{k n}, \quad \delta_{s-1, n}\left(Y_{k n}\right)=0 \quad \text { for } s \leqslant k \leqslant m \text {. }
\end{aligned}
$$

Finally, we get a sequence of Ore extensions: $\mathcal{A}, \mathcal{A}_{11}, \ldots, \mathcal{A}_{1, n-1}, \mathcal{A}_{m n}, \ldots, \mathcal{A}_{2 n}$, and we put $\mathcal{B}=\mathcal{A}_{2 n}$. Iterative applications of Remark 2.5 show that $\mathcal{B}$ can be easily described as the quotient of a free algebra in $m n$ generators. Clearly, we have constructed $\mathcal{B}$ in such a way that the relations between the $m n$ generators of this algebra are exactly the same as those holding between the generators of $\tilde{\boldsymbol{X}}$ as noted in Proposition 2.2. It follows at once that we can define a morphism of algebras

$$
\begin{aligned}
& \varphi: \mathcal{B} \rightarrow \mathbb{K}_{q}[\boldsymbol{X}]_{X_{1 n}}, \\
& Y_{k l}^{\prime} \mapsto X_{k l}^{\prime} \quad \text { for } 2 \leqslant k \leqslant m, \quad 1 \leqslant l \leqslant n-1 \text {, } \\
& Y_{1 n} \mapsto X_{1 n}, \\
& Y_{1 l} \mapsto X_{1 l} \quad \text { for } 1 \leqslant l \leqslant n-1, \\
& Y_{k n} \mapsto X_{k n} \quad \text { for } 2 \leqslant k \leqslant m \text {. }
\end{aligned}
$$

Proposition 2.6. The morphism $\varphi$ is an isomorphism.

Proof. The surjectivity of $\varphi$ is obvious since the entries of $\tilde{\boldsymbol{X}}$ together with $X_{1 n}^{-1}$ form a set of algebra generators for $\mathbb{K}_{q}[\boldsymbol{X}]_{X_{1 n}}$. It remains to prove that $\operatorname{ker} \varphi=\langle 0\rangle$. Recall that $\mathcal{B}$ is obtained from $\mathcal{A}=\mathcal{O}_{q}\left(M_{m-1, n-1}\right)\left[Y_{1 n}^{-1}\right]$ by $(m-1)+(n-1)$ successive Ore extensions that are all extensions of algebras of the type investigated in Lemma 2.3. Thus, we have $\operatorname{GK} \operatorname{dim} \mathcal{B} \leqslant \operatorname{GKdim} \mathcal{A}+(m-1)+(n-1)$. On the other hand, $\mathcal{A}$ is a Laurent polynomial extension of $\mathcal{O}_{q}\left(M_{m-1, n-1}\right)$ thus $\operatorname{GKdim} \mathcal{A}=\operatorname{GKdim} \mathcal{O}_{q}\left(M_{m-1, n-1}\right)+1=$ $(m-1)(n-1)+1$, by [4, Propositions 3.5 and 4.2]. All this together gives

$$
\operatorname{GKdim} \mathcal{B} \leqslant(m-1)(n-1)+1+(m-1)+(n-1)=m n .
$$

On the other hand, we have GKdim $\mathbb{K}_{q}[\boldsymbol{X}]_{X_{1 n}} \geqslant \operatorname{GKdim} \mathbb{K}_{q}[\boldsymbol{X}]=m n$, since $\mathbb{K}_{q}[\boldsymbol{X}]$ is a subalgebra of $\mathbb{K}_{q}[\boldsymbol{X}]_{X_{1 n}}$.

Now, $\mathcal{B}$ is clearly a domain; and so if we assume that $\operatorname{ker} \varphi \neq\langle 0\rangle$, then $\operatorname{GKdim} \mathcal{B} / \operatorname{ker} \varphi<$ $\operatorname{GKdim} \mathcal{B}=m n$. But this is a contradiction since $\mathcal{B} / \operatorname{ker} \varphi \cong \mathbb{K}_{q}[\boldsymbol{X}]_{X_{1 n}}$. Thus, $\varphi$ is injective. 


\section{Reduction of the size of quantum minors}

Using the results of the previous section we are now able to link $k \times k$ quantum minors of $\boldsymbol{X}$, for $k \geqslant 2$, with $(k-1) \times(k-1)$ quantum minors of $\boldsymbol{X}^{\prime}$ (provided $X_{1 n}$ is invertible, of course).

Recall from the introduction that the expression $\left[i_{k}, \ldots, i_{1} \mid j_{1}, \ldots, j_{k}\right]$ stands for a $k \times k$ quantum minor of $\boldsymbol{X}$, namely the quantum determinant of the submatrix of $\boldsymbol{X}$ obtained from $\boldsymbol{X}$ using rows $i_{1}, \ldots, i_{k}$ and columns $j_{1}, \ldots, j_{k}$. This notation is extended to $\boldsymbol{X}^{\prime}$ adding a " $"$ to avoid confusion. Thus, a quantum $k \times k$ minor of $\boldsymbol{X}^{\prime}$ will be expressed by a symbol $\left[i_{k}, \ldots, i_{1} \mid j_{1}, \ldots, j_{k}\right]^{\prime}$. The convention on the index of rows of $\boldsymbol{X}^{\prime}$ (see the introduction to $\S 1$ ) is in order; so, in such an expression, we shall always have $i_{1} \geqslant 2$ and $j_{k} \leqslant n-1$.

The results we need will follow from the special case where $m=n$. In this context, the role played by $(n-1) \times(n-1)$ minors is of special importance; thus we use a more convenient notation for them (coming from [9]). For $1 \leqslant i, j \leqslant n$, the $(n-1) \times(n-1)$ quantum minor of $\boldsymbol{X}$ obtained by deleting the $i$ th row and $j$ th column is denoted $A(i j)$. Moreover, for $2 \leqslant i \leqslant n$ and $1 \leqslant j \leqslant n-1$ we also define $A^{\prime}(i j)$ to be the $(n-2) \times(n-2)$ quantum minor of $\boldsymbol{X}^{\prime}$ obtained from $\boldsymbol{X}^{\prime}$ by deleting the $i$ th row and $j$ th column.

Theorem 3.1. Assume that $m=n$. Then, with the above notation,

$$
\left(\operatorname{det}_{q} \boldsymbol{X}^{\prime}\right) X_{1 n}=X_{1 n}\left(\operatorname{det}_{q} \boldsymbol{X}^{\prime}\right)=(-q)^{1-n} \operatorname{det}_{q} \boldsymbol{X} .
$$

Proof. Note that $\left(\operatorname{det}_{q} \boldsymbol{X}^{\prime}\right) X_{1 n}=X_{1 n}\left(\operatorname{det}_{q} \boldsymbol{X}^{\prime}\right)$ is clear from 2.1. The proof is by induction on $n$. The case where $n=2$ is an obvious consequence of (2.1). We suppose now that the result is true for any integer less than or equal to $n-1$. Because of Lemma 2.1 the relations of [9, Corollary 4.4.4] give us the expansion

$$
\operatorname{det}_{q} \boldsymbol{X}^{\prime}=\sum_{j=1}^{n-1}(-q)^{j-1} X_{2 j}^{\prime} A^{\prime}(2 j) .
$$

Now,

$$
X_{1 n} X_{2 j}^{\prime}=X_{2 j}^{\prime} X_{1 n}=X_{2 j} X_{1 n}-q^{-1} X_{1 j} X_{2 n} \quad \text { for all } j \in\{1, \ldots, n-1\} ;
$$

and so

$$
\begin{aligned}
X_{1 n} \operatorname{det}_{q} \boldsymbol{X}^{\prime} & =\sum_{j=1}^{n-1}(-q)^{j-1} X_{1 n} X_{2 j}^{\prime} A^{\prime}(2 j) \\
& =\sum_{j=1}^{n-1}(-q)^{j-1}\left(X_{2 j} X_{1 n}-q^{-1} X_{1 j} X_{2 n}\right) A^{\prime}(2 j) \\
& =\sum_{j=1}^{n-1}(-q)^{j-1} X_{2 j} X_{1 n} A^{\prime}(2 j)-q^{-1} \sum_{j=1}^{n-1}(-q)^{j-1} X_{1 j} X_{2 n} A^{\prime}(2 j) .
\end{aligned}
$$


By the induction hypotheses we have

$$
X_{1 n} A^{\prime}(2 j)=(-q)^{2-n} A(2 j) \text { for all } j \in\{1, \ldots, n-1\} .
$$

Hence,

$$
\begin{aligned}
X_{1 n} \operatorname{det}_{q} \boldsymbol{X}^{\prime} & =\sum_{j=1}^{n-1}(-q)^{j-1} X_{2 j}(-q)^{2-n} A(2 j)-q^{-1} \sum_{j=1}^{n-1}(-q)^{j-1} X_{1 j} X_{2 n} X_{1 n}^{-1}(-q)^{2-n} A(2 j) \\
& =\sum_{j=1}^{n-1}(-q)^{j+1-n} X_{2 j} A(2 j)-q^{-1} \sum_{j=1}^{n-1}(-q)^{j+1-n} X_{1 j} X_{2 n} X_{1 n}^{-1} A(2 j) .
\end{aligned}
$$

Setting

$$
R=-q^{-1} \sum_{j=1}^{n-1}(-q)^{j+1-n} X_{1 j} X_{2 n} X_{1 n}^{-1} A(2 j),
$$

we obtain

$$
\begin{aligned}
X_{1 n} R & =\sum_{j=1}^{n-1}(-q)^{j-n} X_{1 j} X_{2 n} A(2 j) \\
& =\sum_{j=1}^{n-1}(-q)^{j-n}\left(X_{2 n} X_{1 j}+\left(q-q^{-1}\right) X_{1 n} X_{2 j}\right) A(2 j) \\
& =\sum_{j=1}^{n-1}(-q)^{j-n} X_{2 n} X_{1 j} A(2 j)+\left(q-q^{-1}\right) \sum_{j=1}^{n-1}(-q)^{j-n} X_{1 n} X_{2 j} A(2 j) .
\end{aligned}
$$

On the other hand, from [9, Corollary 4.4.4], we have the relation

$$
\sum_{j=1}^{n}(-q)^{j-2} X_{1 j} A(2 j)=0 .
$$

Thus,

$$
\begin{aligned}
X_{1 n} R=(-q)^{2-n} X_{2 n} \sum_{j=1}^{n-1}(-q)^{j-2} & X_{1 j} A(2 j) \\
& +\left(q-q^{-1}\right)(-q)^{2-n} X_{1 n} \sum_{j=1}^{n-1}(-q)^{j-2} X_{2 j} A(2 j) \\
=(-q)^{2-n} X_{2 n}\left(0-(-q)^{n-2} X_{1 n} A(2 n)\right) & \\
& +\left(q-q^{-1}\right)(-q)^{2-n} X_{1 n} \sum_{j=1}^{n-1}(-q)^{j-2} X_{2 j} A(2 j) .
\end{aligned}
$$

It follows that

$$
R=(-q)^{-1} X_{2 n} A(2 n)+\left(q-q^{-1}\right)(-q)^{2-n} \sum_{j=1}^{n-1}(-q)^{j-2} X_{2 j} A(2 j) .
$$


Thus,

$$
\begin{aligned}
& X_{1 n} \operatorname{det}_{q} \boldsymbol{X}^{\prime} \\
& =\sum_{j=1}^{n-1}(-q)^{j+1-n} X_{2 j} A(2 j)+(-q)^{-1} X_{2 n} A(2 n) \\
& +\left(q-q^{-1}\right)(-q)^{2-n} \sum_{j=1}^{n-1}(-q)^{j-2} X_{2 j} A(2 j) \\
& =(-q)^{2-n} \sum_{j=1}^{n-1}\left((-q)^{j-1}+\left(q-q^{-1}\right)(-q)^{j-2}\right) X_{2 j} A(2 j)+(-q)^{-1} X_{2 n} A(2 n) \\
& =(-q)^{2-n} \sum_{j=1}^{n-1}\left((-q)^{j-1}-(-q)^{j-1}-q^{-1}(-q)^{j-2}\right) X_{2 j} A(2 j)+(-q)^{-1} X_{2 n} A(2 n) \\
& =(-q)^{1-n} \sum_{j=1}^{n-1}(-q)^{j-2} X_{2 j} A(2 j)+(-q)^{1-n}(-q)^{n-2} X_{2 n} A(2 n) \\
& =(-q)^{1-n} \sum_{j=1}^{n}(-q)^{j-2} X_{2 j} A(2 j) .
\end{aligned}
$$

Again, by using [9, Corollary 4.4.4], it follows that $X_{1 n} \operatorname{det}_{q} \boldsymbol{X}^{\prime}=(-q)^{1-n} \operatorname{det}_{q} \boldsymbol{X}$.

Remark 3.2. We thank the referee for pointing out that, when $\mathbb{K}=\mathbb{C}$, Theorem 3.1 follows from [5, Theorem 3.5]. To see this, first notice that, in the notation of [5], there is a surjective morphism of specialization of the indeterminate $q$ of [5] at our scalar $q^{-1}$ :

$$
\begin{aligned}
A_{q}\left(\text { Mat }_{n}\right) & \rightarrow \mathbb{K}_{q}[\boldsymbol{X}], \\
q & \mapsto q^{-1}, \\
t_{i j} & \mapsto X_{i j} .
\end{aligned}
$$

Now, [5, Theorem 3.5] applied with $P=\{1\}$ and $Q=\{n\}$ gives that the $(n-1) \times(n-1)$ matrix $U=\left(t_{1 j} t_{i n}-q^{-1} t_{1 n} t_{i j}\right)=\left(-q^{-1} t_{1 n}\left(t_{i j}-q t_{1 j} t_{i n} t_{1 n}^{-1}\right)\right)$ (with $2 \leqslant i \leqslant n$ and $1 \leqslant j \leqslant n-1)$ is $q^{-1}$-quantum in our terminology and that $\operatorname{det}_{q} U=\left(\operatorname{det}_{q} T\right) t_{1 n}^{n-2}$. Since $X_{1 n}$ commutes with all the entries of $\boldsymbol{X}^{\prime}$, applying the specialization morphism gives us that $\boldsymbol{X}^{\prime}$ is $q$-quantum (the first part of our Lemma 2.1) and that $\left(\operatorname{det}_{q} \boldsymbol{X}^{\prime}\right) X_{1 n}=$ $(-q)^{1-n} \operatorname{det}_{q} \boldsymbol{X}$ (our Theorem 3.1).

By using Theorem 3.1, we can establish Corollary 3.3, which links $(p-1) \times(p-1)$ minors of $\boldsymbol{X}^{\prime}$ with $p \times p$ minors of $\boldsymbol{X}$, for $p \geqslant 2$, that involve the first row and the last column of $\boldsymbol{X}$.

Corollary 3.3. Let $p \geqslant 2$. Suppose that

$$
I=\left\{1=i_{1}<\cdots<i_{p}\right\} \subseteq \mathbb{N}_{m} \quad \text { and } \quad J=\left\{j_{1}<\cdots<j_{p}=n\right\} \subseteq \mathbb{N}_{n}
$$

and set

$$
I^{\prime}=\left\{i_{2}<\cdots<i_{p}\right\} \quad \text { and } \quad J^{\prime}=\left\{j_{1}<\cdots<j_{p-1}\right\}
$$


Then

$$
\left[I^{\prime} \mid J^{\prime}\right]^{\prime}=(-q)^{1-p}[I \mid J] X_{1 n}^{-1}=(-q)^{1-p} X_{1 n}^{-1}[I \mid J] .
$$

Proof. This is an immediate consequence of Theorem 3.1 applied to the square submatrix obtained from $\boldsymbol{X}$ by using rows $i_{1}, \ldots, i_{p}$ and columns $j_{1}, \ldots, j_{p}$ of $\boldsymbol{X}$.

Recall from $\S 1$ that, for $1 \leqslant t \leqslant \min \{m, n\}$, we denote by $\mathcal{I}_{t}(\boldsymbol{X})$ the ideal generated in $\mathbb{K}_{q}[\boldsymbol{X}]$ by the $t \times t$ minors of $\boldsymbol{X}$. Clearly, the ideal generated by the $t \times t$ minors of $\boldsymbol{X}$ in $\mathbb{K}_{q}[\boldsymbol{X}]_{X_{1 n}}$ is just $\tilde{\mathcal{I}}_{t}(\boldsymbol{X}):=\mathcal{I}_{t}(\boldsymbol{X}) \mathbb{K}_{q}[\boldsymbol{X}]_{X_{1 n}}$.

Lemma 3.4. Let $1 \leqslant t \leqslant \min \{m, n\}$.

(i) $\mathcal{I}_{t}(\boldsymbol{X})$ coincides with the right ideal of $\mathbb{K}_{q}[\boldsymbol{X}]$ generated by the $t \times t$ minors of $\boldsymbol{X}$.

(ii) $\tilde{\mathcal{I}}_{t}(\boldsymbol{X})$ coincides with the right ideal of $\mathbb{K}_{q}[\boldsymbol{X}]_{X_{1 n}}$ generated by the $t \times t$ minors $[I \mid J]$ of $\boldsymbol{X}$ such that $1 \in I$ and $n \in J$.

Proof. (i) The case where $m=n$ follows at once from [1, Corollary A.2]. Now, set $s:=\max \{m, n\}$. There is a surjective algebra morphism

$$
\begin{aligned}
\pi: \mathcal{O}_{q}\left(M_{s}\right) & \rightarrow \mathcal{O}_{q}\left(M_{m, n}\right), \\
X_{i j} & \mapsto \begin{cases}X_{i j} & \text { if } i \leqslant m \text { and } j \leqslant n, \\
0 & \text { otherwise },\end{cases}
\end{aligned}
$$

and, for $I, J \subseteq \mathbb{N}_{s}$ such that $|I|=|J|=t$, a $t \times t$ minor $[I \mid J]$ of $\mathcal{O}_{q}\left(M_{s}\right)$ is sent to $[I \mid J]$ if $I \subseteq \mathbb{N}_{m}$ and $J \subseteq \mathbb{N}_{n}$ and is sent to 0 otherwise. It follows that the ideal of $\mathcal{O}_{q}\left(M_{m, n}\right)$ generated by the $t \times t$ minors is the image under $\pi$ of the ideal of $\mathcal{O}_{q}\left(M_{s}\right)$ generated by the $t \times t$ minors. From this, we see that point (i) for arbitrary positive integers $m$ and $n$ follows from the special case $m=n$.

(ii) By part (i), $\tilde{\mathcal{I}}_{t}(\boldsymbol{X})$ coincides with the right ideal of $\mathbb{K}_{q}[\boldsymbol{X}]_{X_{1 n}}$ generated by the $t \times t$ minors. For the purpose of this proof, denote by $\mathcal{S}$ the set of $t \times t$ minors $[I \mid J]$ of $\boldsymbol{X}$ such that $1 \in I$ and $n \in J$.

Let $[I \mid J]$ be a $t \times t$ minor of $\boldsymbol{X}$ such that $1 \in I$ but $n \notin J$. We may apply [9, Corollary 4.4.4] to the subalgebra of $\mathbb{K}_{q}[\boldsymbol{X}]$ generated by those $X_{i j}$ such that $i \in I$ and $j \in J \cup\{n\}$. This leads to the equation

$$
\sum_{j \in J \cup\{n\}}(-q)^{\bullet}\left[I \mid J_{j}\right] X_{1 j}=0,
$$

where, for $j \in J \cup\{n\}$, we put $J_{j}=J \cup\{n\} \backslash\{j\}$, and occurrences of $(-q)^{\bullet}$ denote integer powers of $-q$, which it is not necessary to specify exactly. Since $J_{n}=J$, it follows that the equation

$$
[I \mid J]=-\sum_{j \in J}(-q)^{\bullet}\left[I \mid J_{j}\right] X_{1 j} X_{1 n}^{-1}
$$

holds in $\mathbb{K}_{q}[\boldsymbol{X}]_{X_{1 n}}$. Now, $n \in J_{j}$ for each $j \in J$; and so we have shown that $[I \mid J]$ is in the right ideal generated by $\mathcal{S}$ in $\mathbb{K}_{q}[\boldsymbol{X}]_{X_{1 n}}$. By a similar argument, we prove that a $t \times t$ minor $[I \mid J]$ such that $1 \notin I$ but $n \in J$ is in the right ideal generated by $\mathcal{S}$ in $\mathbb{K}_{q}[\boldsymbol{X}]_{X_{1 n}}$. 
It remains to deal with a $t \times t$ minor $[I \mid J]$ such that $1 \notin I$ and $n \notin J$. In this case, we apply [9, Corollary 4.4.4] in the subalgebra of $\mathbb{K}_{q}[\boldsymbol{X}]$ generated by those $X_{i j}$ such that $i \in I \cup\{1\}$ and $j \in J \cup\{n\}$. This gives us the relation

$$
[I \cup\{1\} \mid J \cup\{n\}]=\sum_{j \in J \cup\{n\}}(-q)^{\bullet}\left[I \mid J_{j}\right] X_{1 j}=\sum_{j \in J}(-q)^{\bullet}\left[I \mid J_{j}\right] X_{1 j}+(-q)^{\bullet}[I \mid J] X_{1 n},
$$

where, for $j \in J \cup\{n\}$, we put $J_{j}=J \cup\{n\} \backslash\{j\}$. Another application of [9, Corollary 4.4.4] gives

$$
[I \cup\{1\} \mid J \cup\{n\}]=\sum_{j \in J \cup\{n\}}(-q)^{\bullet}\left[I \cup\{1\} \backslash\{s\} \mid J_{j}\right] X_{s j},
$$

where $s=\max I$. Equation (3.3) and the results we established above show that the $(t+1) \times(t+1)$ minor $[I \cup\{1\} \mid J \cup\{n\}]$ is in the right ideal generated in $\mathbb{K}_{q}[\boldsymbol{X}]_{X_{1 n}}$ by $\mathcal{S}$. Thus, using (3.2), it follows that $[I \mid J]$ is also in the right ideal generated in $\mathbb{K}_{q}[\boldsymbol{X}]_{X_{1 n}}$ by $\mathcal{S}$. The proof is now complete.

Recall that, by Lemma 2.1 and Proposition 2.4 we know that the subalgebra of $\mathbb{K}_{q}[\boldsymbol{X}]_{X_{1 n}}$ generated by the $X_{i j}^{\prime}$ for $2 \leqslant i \leqslant m$ and $1 \leqslant j \leqslant n-1$ is isomorphic to $\mathcal{O}_{q}\left(M_{m-1, n-1}\right)$ and that we denote it by $\mathbb{K}_{q}\left[\boldsymbol{X}^{\prime}\right]$. Following our conventions, if $2 \leqslant t \leqslant \min \{m, n\}$, we denote by $\mathcal{I}_{t-1}\left(\boldsymbol{X}^{\prime}\right)$ the ideal of $\mathbb{K}_{q}\left[\boldsymbol{X}^{\prime}\right]$ generated by the $(t-1) \times(t-1)$ minors of $\boldsymbol{X}^{\prime}$. In this notation, we have the following important result.

Proposition 3.5. For $2 \leqslant t \leqslant \min \{m, n\}$, the following equality holds:

$$
\tilde{\mathcal{I}}_{t}(\boldsymbol{X})=\mathcal{I}_{t-1}\left(\boldsymbol{X}^{\prime}\right) \mathbb{K}_{q}[\boldsymbol{X}]_{X_{1 n}} .
$$

Proof. By Lemma 3.4 (ii), we know that $\tilde{\mathcal{I}}_{t}(\boldsymbol{X})$ coincides with the right ideal of $\mathbb{K}_{q}[\boldsymbol{X}]_{X_{1 n}}$ generated by the $t \times t$ minors $[I \mid J]$ of $\boldsymbol{X}$ such that $1 \in I$ and $n \in J$. On the other hand, let $[I \mid J]$ be a $t \times t$ minor of $\boldsymbol{X}$ such that $1 \in I$ and $n \in J$. Applying Corollary 3.3, we have $(-q)^{1-t}[I \mid J]=[I \backslash\{1\} \mid J \backslash\{n\}]^{\prime} X_{1 n}$. Thus, $\tilde{\mathcal{I}}_{t}(\boldsymbol{X})$ coincides with the right ideal of $\mathbb{K}_{q}[\boldsymbol{X}]_{X_{1 n}}$ generated by the $(t-1) \times(t-1)$ minors $[I \mid J]^{\prime}$ of $\boldsymbol{X}^{\prime}$ (such that $I \subseteq\{2, \ldots, n\}$ and $J \subseteq\{1, \ldots, m-1\}$ ). On the other hand, Lemma 3.4 (i) shows that $\mathcal{I}_{t-1}\left(\boldsymbol{X}^{\prime}\right)$ is the right ideal of $\mathbb{K}_{q}\left[\boldsymbol{X}^{\prime}\right]$ generated by the $(t-1) \times(t-1)$ minors of $\boldsymbol{X}^{\prime}$; so, the proof is complete.

Recall from the introduction that, for any positive integer $t$ such that $t \leqslant \min \{m, n\}$, we define the quantum determinantal ring $R_{t}(\boldsymbol{X}):=\mathbb{K}_{q}[\boldsymbol{X}] / \mathcal{I}_{t}(\boldsymbol{X})$. This is a domain by $\left[\mathbf{1}\right.$, Corollary 2.6]. If we put $x_{i j}:=X_{i j}+\mathcal{I}_{t}(\boldsymbol{X})$, for $1 \leqslant i \leqslant m$ and $1 \leqslant j \leqslant n$, then there is a canonical isomorphism

$$
R_{t}(\boldsymbol{X})_{x_{1 n}} \cong \mathbb{K}_{q}[\boldsymbol{X}]_{X_{1 n}} / \tilde{\mathcal{I}}_{t}(\boldsymbol{X}) .
$$

We finish this subsection by showing that, for $2 \leqslant t \leqslant \min \{m, n\}$, the ring $R_{t}(\boldsymbol{X})_{x_{1 n}}$ can be described as an iterated Ore extension of $R_{t-1}\left(\boldsymbol{X}^{\prime}\right)$. To achieve this aim, we will have to make use of Proposition 2.6, which shows that $\mathbb{K}_{q}[\boldsymbol{X}]_{X_{1 n}}$ can be obtained from its subalgebra $\mathbb{K}_{q}\left[\boldsymbol{X}^{\prime}\right]$ by iterated Ore extensions adding successively $X_{1 n}^{ \pm 1}, X_{11}, \ldots, X_{1, n-1}, X_{m n}, \ldots, X_{2 n}$. 
Theorem 3.6. For $2 \leqslant t \leqslant \min \{m, n\}$, the ring $R_{t}(\boldsymbol{X})_{x_{1 n}}$ is a localization of an iterated Ore extension of $R_{t-1}\left(\boldsymbol{X}^{\prime}\right)$.

Proof. We start by giving a list of relations between a $(t-1) \times(t-1)$ minor of $\boldsymbol{X}^{\prime}$ and the generators $X_{1 n}^{ \pm 1}, X_{11}, \ldots, X_{1, n-1}, X_{m n}, \ldots, X_{2 n}$. (Note that, by Lemma 2.1, we already know that a $(t-1) \times(t-1)$ minor of $\boldsymbol{X}^{\prime}$ commutes with $X_{1 n}^{ \pm 1}$.)

Let $I^{\prime} \subseteq\{2, \ldots, m\}$ and $J^{\prime} \subseteq\{1, \ldots, n-1\}$ be sets of indices such that $\left|I^{\prime}\right|=\left|J^{\prime}\right|=$ $t-1$. Setting $I=I^{\prime} \cup\{1\}$ and $J=J^{\prime} \cup\{n\}$, Corollary 3.3 shows that $(-q)^{1-t}[I \mid J]=$ $\left[I^{\prime} \mid J^{\prime}\right]^{\prime} X_{1 n}$. On the other hand, [9, Lemma 4.5.1 and Theorem 4.6.1] give the following relations, for $k \in\{2, \ldots, m\}$ and $l \in\{1, \ldots, n-1\}$.

(1) $X_{1 l}[I \mid J]=[I \mid J] X_{1 l}$ if $l \in J^{\prime}$.

(2) $X_{1 l}[I \mid J]-q[I \mid J] X_{1 l}=q\left(q-q^{-1}\right) \sum_{j<l, j \in J^{\prime}}(-q)^{\bullet} X_{1 j}[I \mid J \cup\{l\} \backslash\{j\}]$ if $l \notin J^{\prime}$.

(3) $X_{k n}[I \mid J]=[I \mid J] X_{k n}$ if $k \in I^{\prime}$.

(4) $X_{k n}[I \mid J]-q^{-1}[I \mid J] X_{k n}=q^{-1}\left(q^{-1}-q\right) \sum_{j>k, j \in I^{\prime}}(-q)^{\bullet} X_{j n}[I \cup\{k\} \backslash\{j\} \mid J]$ if $k \notin I^{\prime}$.

(Points (1) and (3) follow from [9, Theorem 4.6.1], point (2) is the first relation of Lemma 4.5.1(1) in [9], point (4) is the second relation of Lemma 4.5.1(3) in [9].) Hence, for $k \in\{2, \ldots, n\}$ and $l \in\{1, \ldots, n-1\}$, Corollary 3.3 gives the following relations.

$\left(1^{\prime}\right) X_{1 l}\left[I^{\prime} \mid J^{\prime}\right]^{\prime}=q^{-1}\left[I^{\prime} \mid J^{\prime}\right]^{\prime} X_{1 l}$ if $l \in J^{\prime}$.

$\left(2^{\prime}\right) X_{1 l}\left[I^{\prime} \mid J^{\prime}\right]^{\prime}-\left[I^{\prime} \mid J^{\prime}\right]^{\prime} X_{1 l}=q\left(q-q^{-1}\right) \sum_{j<l, j \in J^{\prime}}(-q)^{\bullet} X_{1 j}\left[I^{\prime} \mid J^{\prime} \cup\{l\} \backslash\{j\}\right]^{\prime}$ if $l \notin J^{\prime}$.

(3') $X_{k n}\left[I^{\prime} \mid J^{\prime}\right]^{\prime}=q\left[I^{\prime} \mid J^{\prime}\right]^{\prime} X_{k n}$ if $k \in I^{\prime}$.

(4') $X_{k n}\left[I^{\prime} \mid J^{\prime}\right]^{\prime}-\left[I^{\prime} \mid J^{\prime}\right]^{\prime} X_{k n}=q^{-1}\left(q^{-1}-q\right) \sum_{j>k, j \in I^{\prime}}(-q)^{\bullet} X_{j n}\left[I^{\prime} \cup\{k\} \backslash\{j\} \mid J^{\prime}\right]^{\prime}$ if $k \notin I^{\prime}$.

By Proposition 2.6, $\mathbb{K}_{q}[\boldsymbol{X}]_{X_{1 n}}$ can be obtained from its subalgebra $\mathbb{K}_{q}\left[\boldsymbol{X}^{\prime}\right]$ by iterated Ore extensions adding successively $X_{1 n}^{ \pm 1}, X_{11}, \ldots, X_{1, n-1}, X_{m n}, \ldots, X_{2 n}$. The relations $\left(1^{\prime}\right)-$ $\left(4^{\prime}\right)$ together with the fact that $X_{1 n}$ commutes with any element of the subalgebra $\mathbb{K}_{q}\left[\boldsymbol{X}^{\prime}\right]$ show that, at each step of this Ore extension, the ideal of the base algebra generated by the $(t-1) \times(t-1)$ minors of $\boldsymbol{X}^{\prime}$ is invariant under the corresponding skew derivation. It follows that $\mathbb{K}_{q}[\boldsymbol{X}]_{X_{1 n}} / \mathcal{I}_{t-1}\left(\boldsymbol{X}^{\prime}\right) \mathbb{K}_{q}[\boldsymbol{X}]_{X_{1 n}}$ is isomorphic to an iterated Ore extension of $\mathbb{K}_{q}\left[\boldsymbol{X}^{\prime}\right] / \mathcal{I}_{t-1}\left(\boldsymbol{X}^{\prime}\right)$. But Proposition 3.5 shows that $\tilde{\mathcal{I}}_{t}(\boldsymbol{X})=\mathcal{I}_{t-1}\left(\boldsymbol{X}^{\prime}\right) \mathbb{K}_{q}[\boldsymbol{X}]_{X_{1 n}}$. The proof is thus complete.

\section{Quantum determinantal rings are maximal orders}

Let $R$ be a noetherian domain with division ring of fractions $Q$. Then $R$ is said to be a maximal order in $Q$ if the following condition is satisfied: if $T$ is a ring such that $R \subseteq T \subseteq Q$ and such that there exist non-zero elements $a, b \in R$ with $a T b \subseteq R$, then $T=R$. This condition is the natural non-commutative analogue of normality for 
commutative domains (see, for example, $[\mathbf{7}, \S 5.1]$ ). In this section, we investigate the maximal order condition for determinantal rings.

Recall that the quantum determinantal ring $R_{t}(\boldsymbol{X}):=\mathbb{K}_{q}[\boldsymbol{X}] / \mathcal{I}_{t}(\boldsymbol{X})$ is a domain by $\left[\mathbf{1}\right.$, Corollary 2.6]. Also, recall that $R_{2}(\boldsymbol{X})$ has been shown to be a maximal order in $[\mathbf{1 0}]$. In this section, we prove that $R_{t}(\boldsymbol{X})$ is a maximal order in its division ring of fractions, when $\mathbb{K}=\mathbb{C}$ and for $q$ an element of $\mathbb{C}$ transcendental over $\mathbb{Q}$.

The following lemma from [10] (see [10, Lemma 1.1]) is recalled here for the convenience of the reader.

Lemma 4.1. Let $R$ be a noetherian domain and $Q=\operatorname{Frac} R$ its division ring of fractions. Assume there exists a non-zero normal element $x$ in $R$ such that

(1) $x R=\bigcap_{i=1}^{r} \mathfrak{p}_{i}$, where, for $i \in\{1, \ldots, r\}, \mathfrak{p}_{i}$ is a completely prime ideal of $R$; and

(2) the localization $R_{x}$ of $R$ with respect to the set $\left\{x^{i}, i \in \mathbb{N}\right\}$ is a maximal order in $Q$.

Let $\tau$ be the automorphism of $R$ associated with $x$; that is $a x=x \tau(a)$ for all $a \in R$. Suppose that $\tau\left(\mathfrak{p}_{i}\right) \subseteq \mathfrak{p}_{i}$ for $i \in\{1, \ldots, r\}$. Then $R$ is a maximal order in $Q$.

One case where the above result immediately applies is the case in which the ideal $x R$ itself is a completely prime ideal. It is this case that we want to use. The applicability of the above lemma to determinantal rings is a consequence of the results of the previous section, since we can deduce the following result.

Theorem 4.2. Assume that $\mathbb{K}=\mathbb{C}$ and let $q$ be an element of $\mathbb{C}$ transcendental over $\mathbb{Q}$. Let $t$ be an integer such that $0<t \leqslant \min \{m, n\}$. Then $R_{t}(\boldsymbol{X})$ is a maximal order.

Proof. For $t=1$ this is trivial, and for $t=2$ it is [10, Théorème 2.3.11]. We proceed by induction on $t$. Assume that the result is true for an integer $s \geqslant 2$ and set $t=s+1$. By Theorem 3.6, $R_{t}(\boldsymbol{X})_{x_{1 n}}$ is a localization of an iterated Ore extension of $R_{t-1}\left(\boldsymbol{X}^{\prime}\right)$. The induction hypothesis shows that $R_{t-1}\left(\boldsymbol{X}^{\prime}\right)$ is a maximal order in its quotient ring and so $R_{t}(\boldsymbol{X})_{x_{1 n}}$ is a maximal order by [8, V.2.5 and IV.2.1]. However, [6, Corollary 10.7] shows that $\left\langle x_{1 n}\right\rangle$ is a completely prime ideal of $R_{t}(\boldsymbol{X})$. Thus, Lemma 4.1 shows that $R_{t}(\boldsymbol{X})$ is a maximal order.

We conjecture that this result holds for arbitrary non-zero $q$ in any field $\mathbb{K}$ and for all $t$. We conclude by proving that $\mathcal{O}_{q}\left(M_{n}\right) /\left\langle\operatorname{det}_{q}\right\rangle$ is a maximal order. All that remains to be proved after the above discussion is that the ideal $\left\langle\operatorname{det}_{q}, X_{1 n}\right\rangle$ is a completely prime ideal. This is what we do next.

\section{5. $\mathcal{O}_{q}\left(M_{n}\right) /\left\langle\operatorname{det}_{q}\right\rangle$ is a maximal order}

In this section we need to use the preferred bases in $\mathcal{O}_{q}\left(M_{u, v}\right)$ developed in [1] , and we follow the notation of that paper. See, in particular, [1, Corollary 1.11]. We recall the notation $\left[T \mid T^{\prime}\right]$ for the product of quantum minors corresponding to an allowable 
bitableau $\left(T, T^{\prime}\right)$. We recall also that it is sometimes convenient to label rows of $\left(T, T^{\prime}\right)$ in the form $(I, J)$, where $I$ and $J$ are sets of row and column indices, respectively (of course, $I \subseteq\{1, \ldots, u\}$ and $J \subseteq\{1, \ldots, v\}$ ); such a pair is called an index pair (see $[\mathbf{1}, \S 1]$ ). Many of the results in $[\mathbf{1}]$ are stated for the square case $\mathcal{O}_{q}\left(M_{u, u}\right)$, and there are easy extensions to the rectangular case $\mathcal{O}_{q}\left(M_{u, v}\right)$ (see, for example, $[\mathbf{1}, 1.11]$ and $[\mathbf{2}, \S 2]$ for more details of this standard procedure).

We aim to prove that the ideal $\left\langle\operatorname{det}_{q}, X_{1 n}\right\rangle$ of $\mathcal{O}_{q}\left(M_{n}\right)$ is a completely prime ideal for each $n \geqslant 3$ by using the following result of Jordan [3].

Proposition 5.1. Let $\sigma$ be an automorphism and let $\delta$ be a $\sigma$-derivation of a domain $A$. Let $R=A[x ; \sigma, \delta]$. Let $c$ be a normal element of $R$ of the form $d x+e$, where $d, e \in A$ and $d \neq 0$. Let $\beta$ be the automorphism of $R$ such that $c r=\beta(r) c$ for all $r \in R$. Then $\beta(A)=A$, the element $d$ is normal in $A$ and $\beta(a) d=d \sigma(a)$ for all $a \in A$. Furthermore, if $e$ is regular modulo the ideal $A d=d A$, then $R / R c$ is a domain.

Proposition 5.2. The ideal $\left\langle\operatorname{det}_{q}, X_{1 n}\right\rangle$ of $\mathcal{O}_{q}\left(M_{n}\right)$ is a completely prime ideal for each $n \geqslant 3$.

Proof. The idea is first to factor out $X_{1 n}$ obtaining a domain and then to factor out $D_{n}:=\operatorname{det}_{q}$ and see that we still have a domain by invoking Jordan's Domain Theorem. We use the notation from that theorem. Let $T$ be the subalgebra of $\mathcal{O}_{q}\left(M_{n}\right)$ generated by all $X_{i j}$ except $X_{n n}$. Set $A:=T /\left\langle X_{1 n}\right\rangle$. Then $A$ is a domain. Let $R:=A\left[X_{n n} ; \sigma, \delta\right] \cong$ $\mathcal{O}_{q}\left(M_{n}\right) /\left\langle X_{1 n}\right\rangle$. Let $D_{n}$ denote the quantum determinant of $\mathcal{O}_{q}\left(M_{n}\right)$ and let $D_{n-1}$ denote the quantum determinant of the copy of $\mathcal{O}_{q}\left(M_{n-1}\right)$ generated by the $X_{i j}$ with $i, j<n$. Note that $D_{n-1}=A(n n)$ in the notation introduced before Theorem 3.1. Set $x:=$ $X_{n n} \in R$ and $d:=\overline{D_{n-1}} \in A \subseteq R$. Note that $d \neq 0$ in $R$. Finally, set $c:=\overline{D_{n}} \in R$. The quantum Laplace expansion of $D_{n}$ by the $n$th column gives $D_{n}=\sum_{i=1}^{n} \pm q^{\bullet} A($ in $) X_{i n}$ so that $c=d x+e$, where $e$ is the image in $A$ of $\sum_{i=1}^{n-1} \pm q^{\bullet} A(i n) X_{i n} \in T$.

Note that $R /\langle c\rangle \cong \mathcal{O}_{q}\left(M_{n}\right) /\left\langle D_{n}, X_{1 n}\right\rangle$. We show that this is a domain by showing that Jordan's Theorem applies. Note that $c$ is normal (in fact, central) in $R$. All we need to do is to observe that $e$ is regular modulo the ideal $d A=A d$ of the ring $A$. However, $A / d A$ is isomorphic to an iterated Ore extension of $\mathcal{O}_{q}\left(M_{n-1}\right) /\left\langle D_{n-1}\right\rangle$. Now $\mathcal{O}_{q}\left(M_{n-1}\right) /\left\langle D_{n-1}\right\rangle$ is a domain by [1, Theorem 2.5] or by [3, Example 2]; and so $A / d A$ is a domain. Thus, all we have to do is show that $e \notin d A$.

Suppose that $e \in d A$. Then $e=\overline{D_{n-1} \alpha}$ for some $\alpha \in T$. Taking preimages, we obtain

$$
\sum_{i=1}^{n-1} \pm q^{\bullet} A(i n) X_{i n}=A(n n) \alpha+\beta X_{1 n}
$$

for some $\beta \in T$. Now, each term on the left-hand side is an element of $\mathcal{O}_{q}\left(M_{n}\right)$ of bidegree $(1, \ldots, 1 ; 1, \ldots, 1)$ in the $\mathbb{Z}^{n} \times \mathbb{Z}^{n}$ grading of $\mathcal{O}_{q}\left(M_{n}\right)$ described in $[\mathbf{1}, 1.5]$. Hence, we may assume that each term on the right-hand side also has this bidegree. This implies that $\alpha=\lambda X_{n n}$ for some scalar $\lambda \in \mathbb{K}$. Since $X_{n n} \notin T$, this implies that $\alpha=0$. In the resulting equation

$$
\sum_{i=1}^{n-1} \pm q^{\bullet} A(i n) X_{i n}=\beta X_{1 n},
$$


each of the terms on the left-hand side is a preferred product. However, if we write $\beta=\sum \lambda_{i}\left[T_{i} \mid T_{i}^{\prime}\right]$ in terms of the preferred basis, then $\beta X_{1 n}=\sum \lambda_{i}\left[T_{i} \mid T_{i}^{\prime}\right] X_{1 n}$ is again in preferred form. The equation

$$
\sum_{i=1}^{n-1} \pm q^{\bullet} A(i n) X_{i n}=\sum \lambda_{i}\left[T_{i} \mid T_{i}^{\prime}\right] X_{1 n}
$$

then contradicts the independence of the preferred basis.

Thus $e \notin d A$ and we have all of the hypotheses of Jordan's Theorem and conclude that $R /\langle c\rangle \cong \mathcal{O}_{q}\left(M_{n}\right) /\left\langle D_{n}, X_{1 n}\right\rangle$ is a domain, as required.

It is interesting to note that when $n=2$ the above result fails. In this case, the ideal $\left\langle\operatorname{det}_{q}, X_{12}\right\rangle$ is semiprime; in fact, it is the intersection of two completely prime ideals, each of which is fixed by the automorphism determined by $X_{1 n}$, so Lemma 4.1 is applicable. However, this case has already been dealt with in $[\mathbf{1 0}]$.

Theorem 5.3. $\mathcal{O}_{q}\left(M_{n}\right) /\left\langle\operatorname{det}_{q}\right\rangle$ is a maximal order in its division ring of quotients for each $n \geqslant 2$.

Proof. When $n=2$ this is proved in [10, Théorème 2.3.11] (and can be proved directly from Theorem 4.2 by the reasoning in the previous paragraph). An inductive argument similar to that used in Theorem 4.2 finishes the proof.

\section{Appendix A. Some useful relations}

In this section we collect some useful relations. They are essentially derived from results of $[\mathbf{9}]$.

Relation A 1. If

$$
\left(\begin{array}{ll}
a & b \\
c & d
\end{array}\right)
$$

is a $2 \times 2$ quantum matrix, then the following relation holds:

$$
a d-q^{2} d a=\left(1-q^{2}\right)(a d-q b c) .
$$

Relations A 2. If

$$
\left(\begin{array}{lll}
X_{11} & X_{12} & X_{13} \\
X_{21} & X_{22} & X_{23} \\
X_{31} & X_{32} & X_{33}
\end{array}\right)
$$

is a $3 \times 3$ quantum matrix, then the following relations hold.

(1) For $i=2,3$,

(1.1) $X_{11}[i, 1 \mid 1,3]=[i, 1 \mid 1,3] X_{11}($ see $[\mathbf{9},(4.6 .1)])$;

(1.2) $X_{11}[i, 1 \mid 2,3]=q[i, 1 \mid 2,3] X_{11}($ see $[\mathbf{9},(4.5 .1)(1)])$; 
(1.3) $X_{12}[i, 1 \mid 1,3]-q[i, 1 \mid 1,3] X_{12}=\left(q^{-1}-q\right) X_{11}[i, 1 \mid 2,3]($ see $[\mathbf{9},(4.5 .1)(1)])$;

(1.4) $X_{12}[i, 1 \mid 2,3]=[i, 1 \mid 2,3] X_{12}($ see $[\mathbf{9},(4.6 .1)])$.

(2) For $j=1,2$,

(2.1) $X_{33}[3,1 \mid j, 3]=[3,1 \mid j, 3] X_{33}($ see $[\mathbf{9},(4.6 .1)]) ;$

(2.2) $X_{33}[2,1 \mid j, 3]=q^{-1}[2,1 \mid j, 3] X_{33}($ see $[\mathbf{9},(4.5 .1)(3)])$;

(2.3) $X_{23}[3,1 \mid j, 3]-q^{-1}[3,1 \mid j, 3] X_{23}=\left(q-q^{-1}\right) X_{33}[2,1 \mid j, 3]$ (see $\left.[\mathbf{9},(4.5 .1)(3)]\right)$;

(2.4) $X_{23}[2,1 \mid j, 3]=[2,1 \mid j, 3] X_{23}(\operatorname{see}[\mathbf{9},(4.6 .1)])$.

Acknowledgements. This research was partly supported by grants from the Edinburgh and London Mathematical Societies and the European Science Foundation programme 'Noncommutative Geometry'.

\section{References}

1. K. R. Goodearl and T. H. Lenagan, Quantum determinantal ideals, Duke Math. J. 103 (2000), 165-190.

2. K. R. Goodearl, T. H. Lenagan and L. Rigal, The first fundamental theorem of coinvariant theory for the quantum general linear group, Publ. RIMS (Kyoto) 36 (2000), 269-296.

3. D. A. Jordan, Normal elements of degree one in Ore extensions, Commun. Alg. 30 (2002), 803-807.

4. G. R. Krause and T. H. Lenagan, Growth of algebras and Gelfand-Kirillov dimension, Graduate Studies in Mathematics, vol. 22 (American Mathematical Society, Providence, RI, 2000).

5. D. Krob and B. LeClerc, Minor identities for quasi-determinants and quantum determinants, Commun. Math. Phys. 169 (1995), 1-23.

6. S. Launois, Generators for $\mathcal{H}$-invariant prime ideals in $O_{q}\left(\mathcal{M}_{m, p}(\mathbb{C})\right)$, Proc. Edinb. Math. Soc., in press.

7. J. C. McConnell and J. C. Robson, Noncommutative noetherian rings (Wiley, 1987).

8. G. Maury and J. Raynaud, Ordres maximaux au sens de K. Asano, Lecture Notes in Mathematics, vol. 808 (Springer, 1980).

9. B. Parshall and J. Wang, Quantum linear groups, Mem. Am. Math. Soc. 89 (1991), 439.

10. L. Rigal, Normalité de certains anneaux déterminantiels quantiques, Proc. Edinb. Math. Soc. 42 (1999), 621-640. 